\title{
EVALUATION OF BCL-2 EXPRESSION IN CIGARETTE SMOKERS
}

\author{
Shereen Ali *
}

\begin{abstract}
Background: Tobacco use is the most important risk factor linked to oral cancer. Tobacco influences the DNA repair, cell cycle control, proliferation and apoptosis. The present study observed bcl-2 expression to verify its value as prognostic markers in cigarette smokers.

Methods: The study sample comprised 40 smoking males; 20 with hyperplasia and 20 with dysplasia. The duration of smoking and the number of cigarettes were recorded. Hematoxylene and Eosin stained sections were used for histopathologic examination and immunostained sections for evaluation of the expression of bcl-2. The resulting data were analyzed statistically.

Results: The dysplasia group had a higher mean duration of smoking, number of cigarettes per day and total number of cigarettes as well as higher mean area $\%$ and stronger bcl-2 immunoreactions compared to that of the hyperplasia group.
\end{abstract}

Conclusions: Bcl-2 may be a valuable prognostic marker in evaluating the malignant potential of hyperplastic and dysplastic lesions in cigarette smokers.

Keywords: Bcl-2, cigarette smokers, oral cancer

\section{INTRODUCTION}

Tobacco is the most important major risk factor of development of oral cancer ${ }^{(1-4)}$. Diverse tobacco products exist varied from smoking (cigarettes, cigars, pipe) to smokeless tobacco chewing. Cigarettes are the most intensively consumed ${ }^{(2,3)}$.

The effect of tobacco smoke on the oral mucosa results from toxins and carcinogens in the smoke emitted from burning tobacco as well as drying of the mucosa by the high intra-oral temperature and pH change ${ }^{(3)}$.
Cigarette smoke consists of many compounds. Among them, polycyclic compounds (mainly nicotine), aldehydes, arsenic and nickel are considered carcinogenic ${ }^{(5)}$. They affect the DNA repair, cell cycle controls and cause genetic alterations ${ }^{(4)}$. Nicotine stimulates proliferation and impairs apoptosis of epithelial cells, thus predispose to oral cancer development ${ }^{(6,7)}$.

The process of carcinogenesis doesn't involve increased cell proliferation alone but also decreased apoptosis, which allow increased cell survival and

* Lecturer, Department of Oral Medicine and Periodontology, Faculty of Dentistry, Cairo University, Lecturer, Department of Oral Medicine, Periodontology and Oral Radiology, Faculty of Dentistry, Misr International University (MIU). 
accumulation of genetic damage ${ }^{(8)}$. Apoptosis is controlled by various proteins including bcl-2. Bcl2 is anti-apoptotic protein regulates the apoptotic pathway by blocking post-mitotic differentiation from apoptosis, consequently inhibits apoptosis ${ }^{(9,10)}$.

The current study was undertaken to observe the expression of bcl-2 and verify its value as prognostic markers in cigarette smokers.

\section{MATERIAL AND METHODS}

\section{Study design}

Observational case-control study.

\section{Study setting and participants}

Enrolled individuals were recruited from both the Diagnostic Center and the Out-Patient Clinic of Oral Medicine, Periodontology and Diagnosis Department, Faculty of Dentistry, Cairo University.

All of them fulfilled the following eligibility criteria. Inclusion criteria included males; cigarette smokers suffering from smoker keratosis; cigarette smokers undergoing minor surgical procedure; individuals who agreed for undergoing the biopsy and systemically free individuals. Exclusion criteria included oral lesions other than smoker's keratosis and smoker's melanosis; forms of tobacco consumption other than cigarettes as well as individuals with sharp, broken teeth or prosthetics and prominent cusps.

The study sample comprised forty smoking males; twenty with hyperplasia and twenty with dysplasia. Sample size calculation was done using R statistical package, version 2.15.2 [Copyright (C) 2012 - The R Foundation for Statistical Computing]. Paired $t$ test power calculation was used to detect the proper sample size. Means and standard deviations were determined according to Garewal et al. (11) which showed that the first study group had a mean bcl-2 expression of $9.35(\mathrm{SD}=2.28)$ and the second group had a mean of $3.56(\mathrm{SD}=0.91)$. The sample size calculation was based on a mean difference of 1.4 points and a pooled standard deviation of the two means' standard deviations. The results showed that a total sample size of 40 participants will be adequate to detect a mean difference between study groups of 1.5 points of Bcl-2 expression (pooled $\mathrm{SD}=1.26$ ) with a power of $90 \%$ and a two-sided significance level of $5 \%$; with equal allocation to two arms.

The patients were enrolled in consecutive order of seeking the clinic to avoid selection bias. For each individual a full history was obtained followed by thorough clinical examination. All teeth opposite to the occlusal plane were examined to detect the presence of any sharp tooth edge, to ensure that no local factor played a role in the changes produced. The number of cigarettes smoked per day and duration of smoking were recorded. The total number of cigarettes was calculated using number of cigarettes smoked per day and duration of smoking. After full description of the study and the entire sample procedure to the enrolled individuals an approved written consent was obtained. This research followed Declaration of Helsinki and was approved by Faculty Research Ethics committee of the Faculty of Oral \& Dental Medicine, Cairo University in September 2016 (number: 16-9-23).

\section{Biopsy procedure}

Oral mucosal tissues $(0.5 \times 0.5 \mathrm{~cm})$ were obtained during minor surgical procedures to which the individual were already subjected. A double wedge small incisional biopsy $(0.5 \times 0.5 \mathrm{~cm})$ was taken from the lesions of smokers suffering from smoker keratosis.

Biopsy material was immediately fixed in $10 \%$ formalin then processed for preparing a paraffin block. Five $\mu \mathrm{m}$ tissue sections were then cut, stained with conventional Hematoxyline \& Eosin (H\&E) for histopathologic diagnosis and immunostained using Cell and Tissue staining kit "Horseraddish 
peroxidase - 3-3' diaminobenzidine (HRP-DAB) system, mouse kit", Mouse-Monoclonal Anti-human bcl-2 Antibody [R\&D systems, Inc. Minneapolis, USA] for evaluation of bcl-2 expression.

\section{Computer image analysis}

The immunostained sections were examined in Oral Pathology department, Faculty of Dentistry, Cairo University by the image analyzer computer system using the software Leica Q Win 515 system (England). The immunoreactivity for bcl-2 in the epithelium was measured in the form of area percentage (area \%).

\section{Statistical analysis}

The data were described as means and standard deviations. The Shapiro-Wilk test was applied to test the normality of the data. Normally distributed data required using the parametric paired Student's t-test to assess the differences between the two groups. Data that was not normally distributed required using the non-parametric Mann-Whitney $\mathrm{U}$ test. The significance level was verified at $\mathrm{P} \leq 0.05$. The results are considered to be statistically significant if p-value was less than 0.05 . R statistical package, version 2.15.2 was used for analyzing the data [Copyright (C) 2012 - The R Foundation for Statistical Computing].

\section{RESULTS}

Table (1) and Figures (1-4) represent the comparison and statistical analysis regarding all the variables.

Histopathological examination of group I revealed: regular stratification of the epithelium with no cellular atypia, intact basement membrane, acanthosis and thick keratin layer. H\&E stained sections of group II showed irregular epithelial stratification as well as architectural disturbance and cytological atypia associated with dysplasia, extending the full thickness of the epithelium in some sections (Figure 5).

Regarding duration and dose of cigarette smoking, the dysplasia group had a higher mean duration of smoking and number of cigarettes per day compared to that of the hyperplasia group with a statistically significant mean difference. In addition, the dysplasia group had a higher mean total number of cigarettes than the hyperplasia group with a statistically significant mean difference.

As regards immunohistochemical analysis, tissue sections of group I gave mild to mode rate immunopositive reaction for bcl-2, where those of group II showed more intense immunoreactions (Figure 5).

Concerning the area\% of bcl-2, the dysplasia group had a higher mean area \% of bcl-2 than the hyperplasia group with a statistically significant mean difference.

TABLE (1) Comparisons between the two studied groups.

\begin{tabular}{|c|c|c|c|c|}
\hline & Hyperplasia & Dysplasia & \multirow{2}{*}{$\begin{array}{c}\text { Mean Difference } \\
( \pm \text { SD })\end{array}$} & Students' $t$ test \\
\cline { 2 - 3 } & Mean $( \pm$ SD) & Mean $( \pm$ SD) & Palue* \\
\hline Age (years) & $41.9( \pm 6.08)$ & $44.7( \pm 6.99)$ & $-2.8( \pm 10.21)$ & $0.3586^{* *}$ \\
\hline Area \% of bcl-2 & $43.87( \pm 5.93)$ & $68.69( \pm 3.63)$ & $-24.82( \pm 7.83)$ & $<0.0001$ \\
\hline Duration of smoking (years) & $18.4( \pm 5.89)$ & $27( \pm 6.18)$ & $-8.6( \pm 9.9)$ & 0.0226 \\
\hline Number of cigarettes per day & $30.5( \pm 7.98)$ & $44.5( \pm 14.42)$ & $-14( \pm 13.7)$ & $0.0316^{* *}$ \\
\hline Total number of cigarettes & $\begin{array}{c}196552.5 \\
( \pm 58197.19)\end{array}$ & $\begin{array}{c}435810 \\
( \pm 184187.02)\end{array}$ & $\begin{array}{c}-239257.5 \\
( \pm 209569.64)\end{array}$ & $0.0019^{* *}$ \\
\hline
\end{tabular}




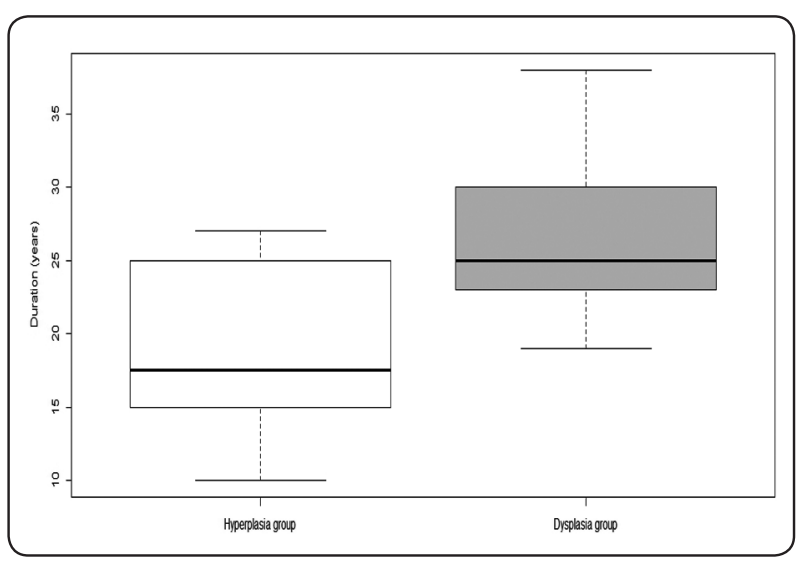

Fig. (1) Comparison of duration of smoking

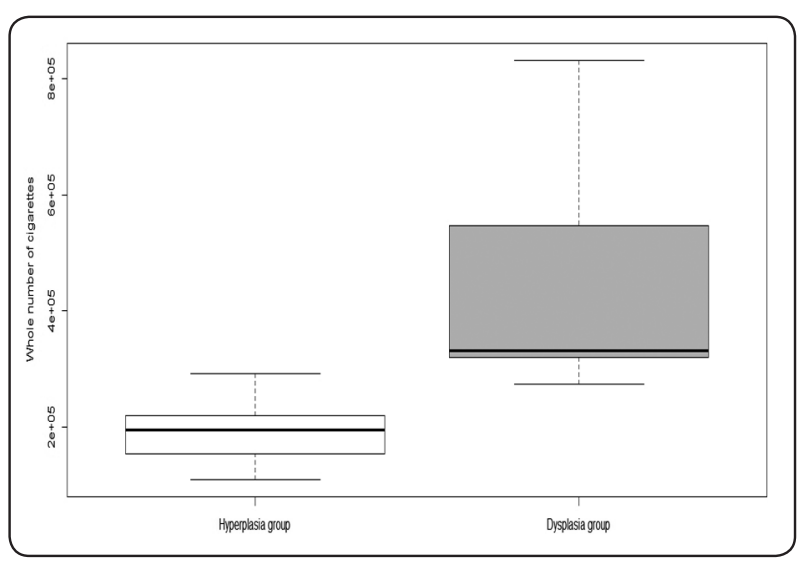

Fig. (3) Comparison of total number of cigarettes

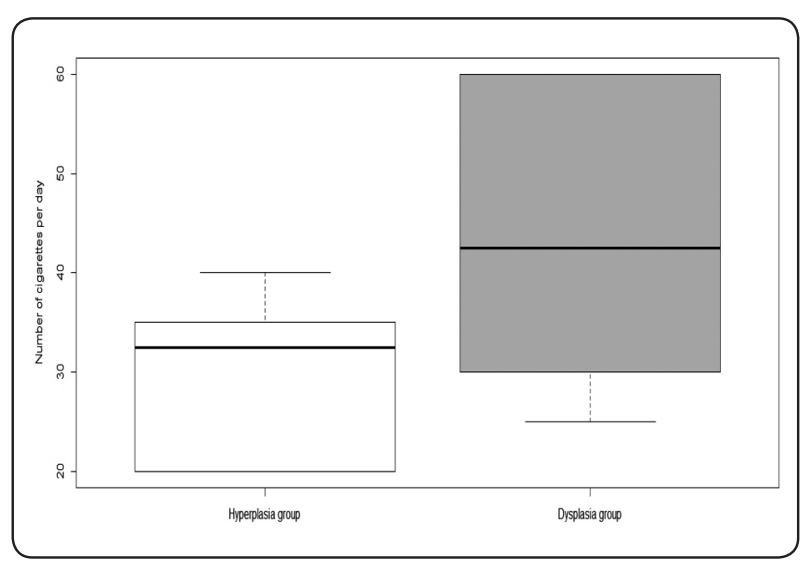

Fig. (2) Comparison of dose of cigarettes per day

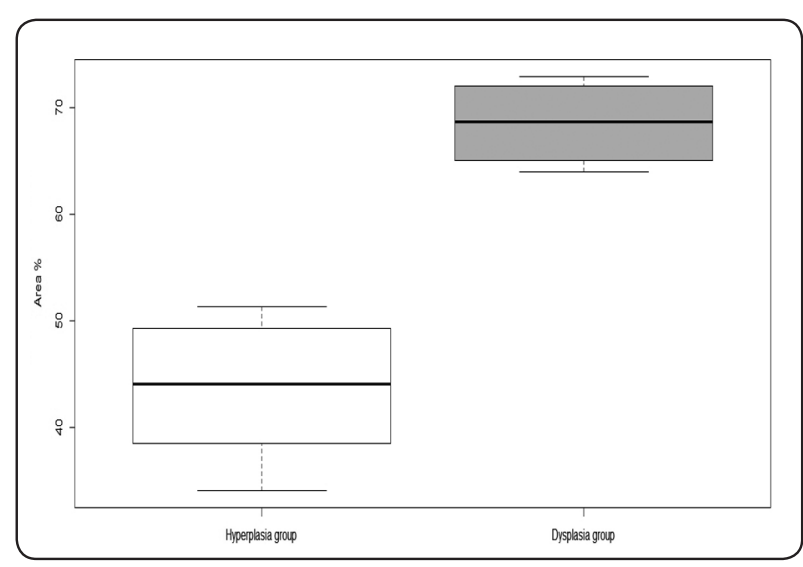

Fig. (4) Comparison of area \% of bcl-2

epithelium protects the underlying tissues from carcinogens including tobacco. One of these protective mechanisms and adaptive responses is upregulation of epithelial proliferation which is revealed as epithelial hyperplasia ${ }^{(14,15)}$. Nicotine impairs apoptosis, which is a defense mechanism to eliminate cancer cells. Thus, deregulation of apoptosis can contribute to oral cancer development ${ }^{(6)}$.

On comparing the number of cigarettes smoked per day and duration of smoking separately, our clinical results revealed statistical significant loss [desquamation and apoptosis] ${ }^{(13)}$. The oral 

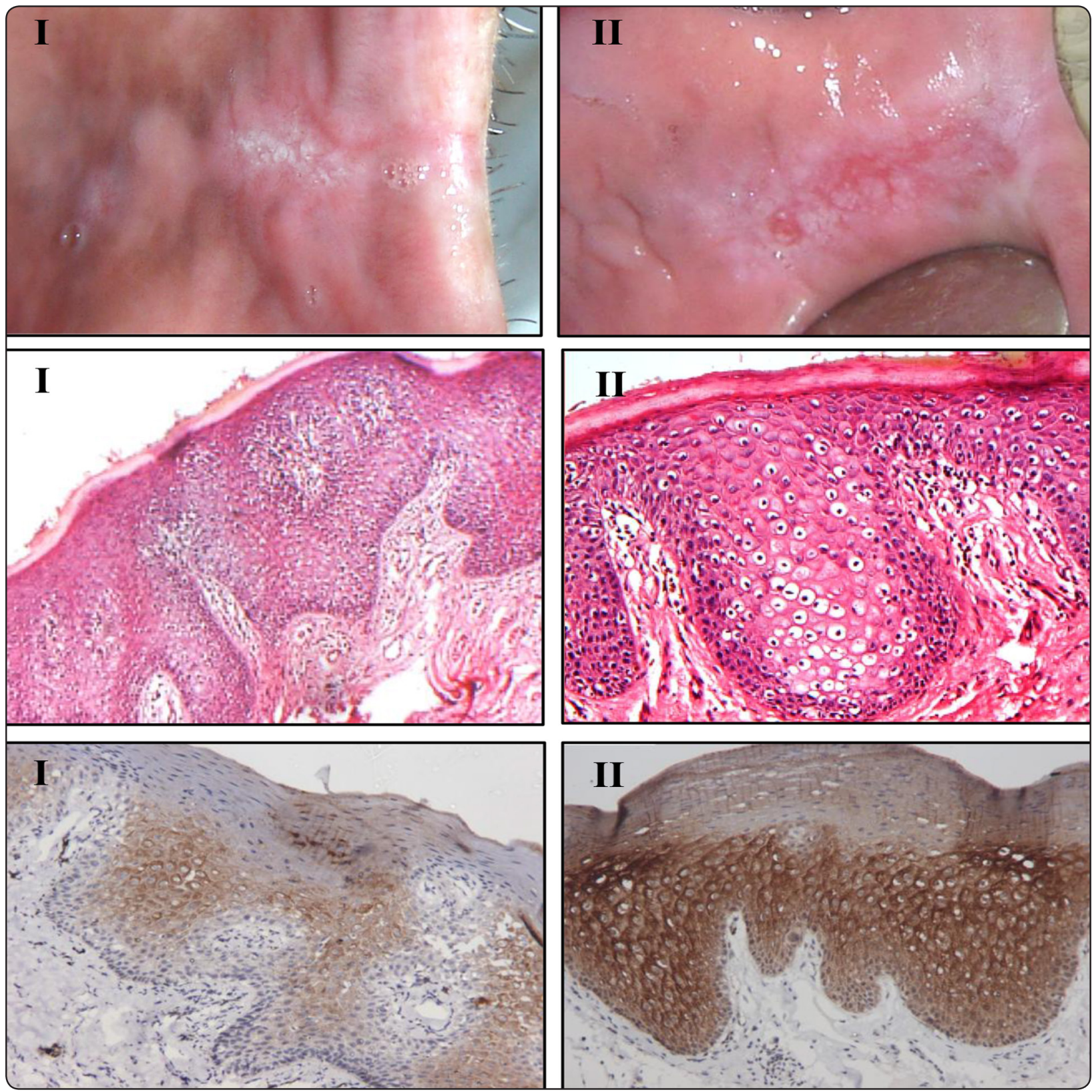

Fig. (5) Clinical photographs and Photomicrographs [H \& E and immunostaining]

difference with higher means in smokers suffering of dysplasia. While, comparing the total number of cigarettes showed a higher statistical significant difference which means that both dose and duration of smoking has combined role in the development of dysplastic changes and malignant transformation in smokers.
This was in agreement with Taybos (2003) who stated that tobacco smoke causes harmful changes in the oral mucosa depend on both, severity and duration of the insult ${ }^{(16)}$. Our results were similar to Souto et al. (2010) who reported that tobacco use has cumulative effect with a trend toward increase in percentage of aneuploid nuclei with longer 
periods of smoking and larger amount of cigarettes consumed $^{(4)}$. Winn (2001) demonstrated that higher risks of oral cancer development were associated with greater amounts of tobacco used and longer duration of use ${ }^{(1)}$.

Another clinicopathological observation was the presence of histopathological changes despite the absence of visible white keratotic lesion.

These were similar to Einstein and Sivapathasundharam (2005); Khandelwal and Solomon (2010); Souto et al. (2010) who revealed cytomorphological changes in buccal epithelial cells of clinically appearing normal mucosa in tobacco users ${ }^{(4,17,18)}$. These were explained by Ten Cate (2003) who stated that the thickness of epithelium affects its ability to evenly reflect the visible light spectrum. Accordingly, not every hyperplastic epithelium is able to block the light reflected from the hemoglobin in the blood vessels of the connective tissue to the observer's eye. Therefore, absence of clinical white lesion doesn't mean that this mucosa is not at risk of malignant transformation ${ }^{(14)}$.

Previous studies confirmed changes in the turnover rate of the oral epithelium and cytomorphological changes in exfoliated epithelial cells in females due to variation in estrogen and progesterone levels ${ }^{(19)}$, for this reason only male patients were enrolled in this study.

At the molecular level, tobacco smoke carcinogens cause genome damage and mutations, in the form of oncogenes activation and alteration of genes regulating apoptosis which generate altered gene products with loss of their regulatory function resulting in dysplastic features ${ }^{(20)}$.

The immunohistochemical results of the current study demonstrated immunopositive reaction of bcl-2 in all smokers. The dysplastic epithelium had stronger immunoreactions with higher mean area $\%$ that revealed statistical significant difference when compared to group I.
These results were consistent with previous studies that evaluated expression of bcl-2 in oral precancer and dysplasia ${ }^{(8,9,21-25)}$. Nair et al. (2011) demonstrated moderate to intense reactivity involving the entire thickness of the epithelium in oral dysplasia ${ }^{(8)}$. Arya et al. (2016) reported positive immunoreactivity in oral precancer lesion involving the basal and parabasal layers ${ }^{(25)}$. Sadiq et al. (2015) confirmed the positive reactivity and revealed statistically significant progressive increase in the count of bcl-2 positive cells from mild, moderate to sever dysplasia ${ }^{(24)}$. In their animal study, Ortega et al. (2016) found that overexpression of bcl-2 was associated with early neoplastic changes in 4-nitroquinoline 1-oxide-induced oral cancer ${ }^{(26)}$.

These results support the notion that bcl2 has important role in the initial steps of oral carcinogenesis. Bcl-2 protein prevents apoptosis, thus its overexpression helps cells to escape apoptosis leading to prolonged cell survival which increase the opportunity of accumulation of mutations that consequently enhance the potential of malignant transformation ${ }^{(8,9,23)}$.

Juneja et al. (2015) and Sadiq et al. (2015) found overexpression of both bcl-2 and $\mathrm{P}_{53}$ in epithelial dysplasia and correlation with the epithelial dysplasia degree ${ }^{(9,24)}$. Sadiq et al. (2015) added that bcl-2 is a better diagnostic marker than $\mathrm{P}_{53}{ }^{(24)}$. Vincenti et al. (2006) mentioned that bcl-2 gives resistance to therapy by blocking treatment-related apoptosis ${ }^{(27)}$.

From the findings in the present study, explanation of changes of the oral epithelium attributed to tobacco smoke could be as follows:

On the cellular level, exposure to tobacco carcinogens result in up-regulation of epithelial proliferation (epithelial hyperplasia) and downregulation of apoptosis. Both cellular events promote oral cancer development. The effect of tobacco is cumulative depending on number of smoked cigarettes together with duration of smoking. On the 
molecular level, tobacco consumption is associated with alteration in proteins regulating apoptosis including bcl-2, where overexpression of bcl-2 is seen in tobacco users with more intense reactivity and larger area percentage in epithelial dysplasia.

Clinical examination is not sufficient for screening of oral cancer in tobacco users, because clinically appearing normal mucosa doesn't confirm that there are no histopathological changes or that this mucosa is not at risk of malignant transformation. Histopathological examination confirms the presence of histopathological changes, however it can't predict the potential for future dysplastic or malignant changes, as not every dysplasia will progress to cancer. Even histologically normal appearing mucosa may have high potential for malignant transformation which can't be shown during histopathological examination. Identifying molecular alterations can help in exploring the potential for malignant transformation.

\section{CONCLUSION}

- In cigarette smokers, the dysplastic epithelium had stronger immunopositive reaction with higher mean area \% of bcl-2 than hyperplastic epithelium.

- Bcl-2 may be a valuable prognostic marker in evaluating the malignant potential of hyperplastic and dysplastic lesions in cigarette smokers.

- There is a trend for association of the appearance of dysplasia and increased consumption of cigarettes as well as longer duration of smoking.

- In cigarette smokers, even clinically appearing normal mucosa may be at risk of malignant transformation.

\section{ACKNOWLEDGEMENTS}

The author would like to thank Dr. Sara Ahmed for her help in the immunohistochemical analysis and Dr. Attera Nazeer for her help in the statistical analysis.

\section{REFERENCES}

1. Winn DM. Tobacco use and oral disease. J Dent Educ 2001; 65(4): 306-12.

2. Kuper H, Adami HO, Boffetta P. Tobacco use and cancer causation: association by tumour type. J Intern Med 2002; 252: 206-24.

3. Sham A, Cheung L, Jin L, Corbet E. The effects of tobacco use on oral health. Hong Kong Med J 2003; 9: 271-7.

4. Souto GR, Caliari MV, Lins CE, de Aguiar MC, de Abreu MH, Mesquita RA. Tobacco use increase the number of aneuploid nuclei in the clinically healthy oral epithelium. $\mathrm{J}$ Oral Pathol Med 2010; 39: 605-10.

5. Vellappally S, Fiala Z, Smejkalova J, Jacob V, Somanathan R. Smoking related systemic and oral diseases. Acta Medica 2007; 50(3): 161-6.

6. Argentin G, Cicchetti R. Genotoxic and antiapoptotic effect of nicotine on human gingival fibroblasts. Toxicol Sci 2004; 79: 75-81.

7. Lee HJ, Guo HY, Lee SK, et al. Effects of nicotine on proliferation, cell cycle, and differentiation in immortalized and malignant oral keratinocytes. J Oral Pathol Med 2005; 34: 436-43.

8. Nair RG, Shameena PM, Varghese I, Sudha S. Immunohistochemical evaluation of bcl-2 oncoprotein in oral dysplasia and carcinoma. OMPJ 2011; 2 (1): 83-8.

9. Juneja S, Chaitanya NB, Agarwal M. Immunohistochemical expression of Bcl-2 in oral epithelial dysplasia and oral squamous cell carcinoma. Indian J Cancer 2015; 52 (4): 505-10.

10. Kumamoto H, Ooya K. Immunohistochemical analysis of bcl-2 family proteins in benign and malignant ameloblastomas. J Oral Pathol Med 1999; 28: 343-9.

11. Garewal J, Garewal R, Sircar K. Expression of Bcl-2 and MIB-1 Markers in Oral Squamous Cell Carcinoma (OSCC) - A Comparative Study. J Clin Diagn Res 2014; 8(7): QC01-QC04.

12. Brunotto M, Zárate A, Cismondi A, Fernández M, Halac R. Valuation of exfoliative cytology as prediction factor in oral mucosa lesions. Med Oral Patol Oral Cir Bucal 2005; 10 (2): 92-102.

13. Squier CA, Kremer MJ. Biology of oral mucosa and esophagus. J Natl Cancer Inst Monogr 2001; 29: 7-15. 
14. Ten Cate AR. Oral histology, development, structure and function. Mosby Company, 2003: 329-75.

15. Karthikeyan R, Reddy BS, Sherlin HJ, et al. Stromal changes in apparently normal mucosa of smokers and pan chewers - a multiparametric approach. Braz J Oral Sci 2008; 7(26): 1602-8.

16. Taybos G. Oral changes associated with tobacco use. Am J Med Sci 2003; 326: 179-82.

17. Einstein TB, Sivapathasundharam B. Cytomorphometric analysis of the buccal mucosa of tobacco users. Indian $\mathrm{J}$ Dent Res 2005; 16(2): 42-6.

18. Khandelwal S, Solomon MC. Cytomorphological Analysis of Keratinocytes in Oral Smears from Tobacco Users and Oral Squamous Cell Carcinoma Lesions - A Histochemical Approach. Int J Oral Sci 2010; 2(1): 45-52.

19. Donald PM, George R, Sriram G, Kavitha B, Sivapathasundharam B. Hormonal changes in exfoliated normal buccal mucosal cells. J Cytol 2013; 30(4): 252-6.

20. Ram H, Sarkar J, Kumar H, Konwar R, Bhatt M, Mohammad S. Oral Cancer: Risk Factors and Molecular Pathogenesis. J Maxillofac Oral Surg 2011; 10(2):132-7.

21. Singh BB, Chandler FW Jr, Whitaker SB, Forbes-Nelson AE. Immunohistochemical evaluation of bcl-2 oncoprotein in oral dysplasia and carcinoma. Oral Surg Oral Med Oral Pathol Oral Radiol Endod 1998; 85(6): 692-8.
22. Sulkowska M, Famulski W, Chyczewski L, Sulkowski S. Evaluation of p53 and bcl-2 oncoprotein expression in precancerous lesions of the oral cavity. Neoplasma 2001; 48(2): $94-8$.

23. Sudha VM, Hemavathy S. Role of bcl-2 oncoprotein in oral potentially malignant disorders and squamous cell carcinoma: An immunohistochemical study. Indian J Dent Res 2011; 22: 520-5.

24. Sadiq H, Ahuja P, Gupta P, Singh G, Singh N, Anand P. Immunohistochemical expression of P53 and Bcl-2 in varying grades of oral epithelial dysplasia. J Pre clin Dent Res 2015; 2 (4): 17-25.

25. Arya V, Singh S, Daniel MJ. Clinicopathological correlation of Bcl-2 oncoprotein expression in oral precancer and cancer. J Oral Biol Craniofac Res 2016; 6:18-23.

26. Ortega A, Goncalves V, Guimaraes M, Junior CR, Spolidorio LC. Overexpression of Bcl-2, SOCS 1, 3 and Cdh 1, 2 are associated with the early neoplasic changes in modified 4-nitroquinoline 1-oxide-induced murine oral cancer model. J Oral Pathol Med 2016; 45: 573-80.

27. De Vicente JC, Olay S, Lequerica-Fernandez P, SanchezMayoral J, Junquera LM, Fresno MF: Expression of Bcl-2 but not Bax has a prognostic significance in tongue carcinoma. J Oral Pathol Med 2006; 35: 140-5. 